\title{
Anterior-inferior plating of middle-third fractures of the clavicle
}

\author{
Chin-En Chen $\cdot$ Rei-Jahn Juhn $\cdot$ Jih-Yang Ko
}

Received: 14 February 2009 / Published online: 10 November 2009

(C) The Author(s) 2009. This article is published with open access at Springerlink.com

\begin{abstract}
Introduction Various techniques have been reported for the treatment of middle-third clavicle (collar bone) fractures. This prospective study was conducted to evaluate the results of anterior-inferior plating using a $3.5-\mathrm{mm}$ reconstruction plate for the treatment of middle-third clavicle fractures.

Patients and methods Twenty-six patients with middlethird clavicle fractures were treated with anterior-inferior plating. The indications for surgery included complete displacement, severe comminution, and marked shortening of the clavicle $(>2 \mathrm{~cm})$.

Results The mean time to union was 14 weeks (range 8-20 weeks). At the time of latest follow-up, all of the patients had returned to their pre-injury activity level. The plates were removed in ten patients after the fractures healed. No patients required plate removal due to implantrelated problems.

Conclusion Anterior-inferior plating is an effective treatment modality for middle-third clavicle fractures, with few complications and early return of shoulder function. The procedure provides stable fixation, avoids risk to vital
\end{abstract}

C.-E. Chen $(\varangle) \cdot$ R.-J. Juhn

Department of Orthopedic Surgery, Golden Hospital,

12-2, Minsheng E. Rd., Pingtung 900, Taiwan, ROC

e-mail: chinenmd@ms21.hinet.net

J.-Y. Ko

Department of Orthopedic Surgery,

Chang Gung Memorial Hospital-Kaohsiung Medical Center,

Kaohsiung, Taiwan, ROC

J.-Y. Ko

Chang Gung University College of Medicine,

Taoyuan, Taiwan, ROC structures below the clavicle, and is associated with a low rate of implant-prominence problems.

Keywords Anterior-inferior plating - Superior plating . Clavicle $\cdot$ Collar bone $\cdot$ Clavicle fracture

\section{Introduction}

Fractures of the clavicle are common in adults, with middle-third fractures accounting for $76 \%$ of cases in one large series [12]. Although most middle-third clavicle fractures heal well with nonoperative treatment, some require surgical intervention. Indications for surgery include severe displacement, more than $2 \mathrm{~cm}$ of clavicle shortening, and severe comminution; these types of fractures place patients at a risk of unsatisfactory outcomes with nonoperative treatment [13]. In one meta-analysis review, open reduction and internal fixation resulted in a nonunion rate of $2.5 \%$, compared with $5.9 \%$ for a closed reduction and sling group [15].

A variety of surgical techniques have been described for the treatment of middle-third clavicle fracture, including plating, Kirschner-wire fixation, knowles-pin fixation, and elastic intramedullary nailing [11]. Despite the popularity of plating clavicle fractures, optimizing fixation remains problematic. Hardware failure may occur, including plate bending, plate breakage or screw pullout. The superior aspect of the clavicle is the load-bearing side, but superior plate prominence can cause patient discomfort, requiring implant removal after the fracture heals. Anterior-inferior plating for middle-third clavicle nonunion has been reported with satisfactory results [9]. This article reports the clinical results of treatment of 26 acute middle-third fractures of the clavicle with anterior-inferior reconstruction plate fixation. 


\section{Patients and methods}

This prospective study involved 26 patients with middle-third fractures of the clavicle who were treated with anterior-inferior $3.5-\mathrm{mm}$ reconstruction plate fixation. The patients underwent treatment at our institution between September 2006 and August 2007. The indications for this technique included complete displacement, severe comminution and marked shortening of the clavicle $(>2 \mathrm{~cm})$. To simplify the study, patients with open fractures and multiple fractures were excluded, as were patients with multiple injuries. Of the 26 patients, 14 were men and 12 were women. The mean age at the time of surgery was 45 years (range 20-76 years). Twenty-one fractures were caused by traffic accidents, and five resulted from falls. The left clavicle was affected in 10 patients and the right clavicle in 16 patients. Preoperative radiographs were assessed for fracture pattern and degree of comminution. According to the AO classification [10], there were $6 \mathrm{~B} 1$ fractures, 19 B2 fractures and 1 B3 fracture (Fig. 1).

Each patient in the study was scheduled for surgery either the same day of the injury or within $48 \mathrm{~h}$. All of the procedures were performed by one senior physician (C.E.C.). Under general anesthesia, the patient was placed in the supine position, and the affected shoulder was elevated with a towel. The affected upper extremity was draped free to allow manipulation in a sterile manner. An incision parallel to the inferior border of the clavicle was made, and dissection was carried down to the fracture site, followed by careful subperiosteal dissection. The fracture was reduced and stabilized temporarily with wire or Kirschner-wire. For comminuted or spiral fractures, wiring the fragments with 0.8 -mm wire was usually done before the application of the reconstruction plate. Because the wiring of fragments might endanger the structures underneath the clavicle, the numbers of the wire use was minimized in correlation to the fracture pattern. One wire was usually used in B1 fractures. For B2 fractures, two wires were used. Three wires were used in B3 fractures. Only if the stability of the clavicle could not be maintained with wiring then one or two Kirschner-wires should be used temporarily to maintain the alignment of the clavicle. Although lag screw may strengthen the stability in some cases, however, the screw may prevent the placement of the reconstruction plate and, therefore, should never be used. An adequate length of 3.5-mm reconstruction plate was contoured with bending irons for application to the curve of the anterior-inferior border of the clavicle (Fig. 2). A minimum purchase in six cortices with screws for proximal fracture end and six cortices for distal fracture end were necessary; otherwise, strengthening the construct with additional wiring was mandatory. In this particular situation, a wire passer was used to protect the structure underneath the clavicle, while the wire was passed through under the clavicle. One 1.0-mm wire was used to strengthen the construct. Cortical screws were preferred for fixation; however, one or two cancellous screws sometimes were used for the distal fracture end, especially in patient more than 60 years of age. The reconstruction plate can always be contoured in one plane along the anterior-inferior border of the clavicle, that way it would make the plating easier and safer. In most cases, a six- to eight-hole reconstruction plate fit well when contoured into an S-shape as viewed on edge and without a bend as viewed with the screw holes in plain sight (Fig. 3). None of the patients received ancillary cancellous bone graft. After fixation, the upper extremity was taken through a gentle range of motion to ensure adequate stability. A drain was placed, and the wound was then closed.

Postoperatively, the involved upper extremity was placed in a sling for comfort. Physical therapy was initiated on the first postoperative day with gentle active-assisted Codman's and pendulum exercise. To minimize the possible scarring problems, taping of the surgical wound with cosmetic strips for 3 months after the stitches were removed and the wound was clear was recommended. The patients were advised to continue wearing the sling for
Fig. 1 The AO classification for diaphyseal fracture of the clavicle

\section{Diaphysis, noncomminuted (B1)} 1.Spiral (B1.1)

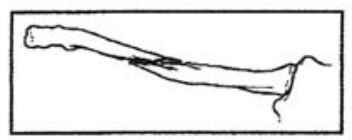

2. Oblique (B1.2)

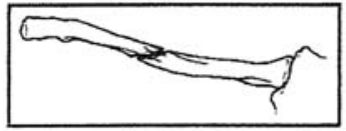

3. Transverse (B1.3)

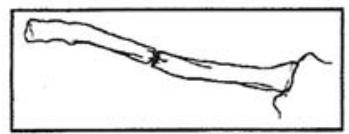

Diaphysis, wedge (B2) 1. Spiral wedge (B2.1)

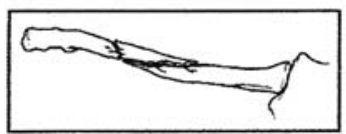

2. Bending wedge (B2.2)

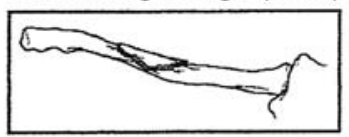

3. Comminuted (B2.3)

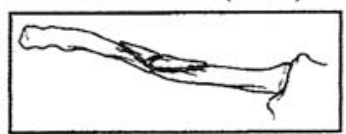

Diaphysis, segmental (B3)

1. Spiral (B3.1)

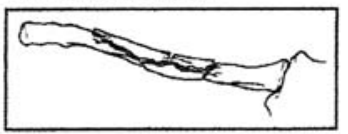

2. 2 transverse (B3.2)

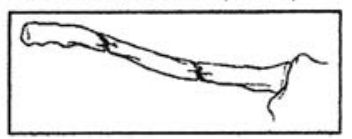

3. Complex comminuted(B3.3)

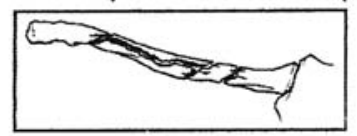




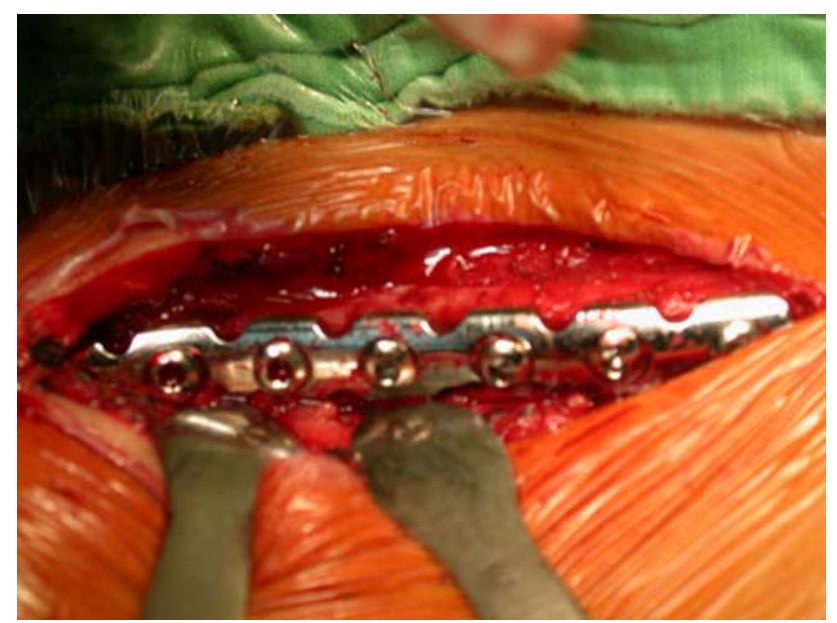

Fig. 2 Anterior-inferior plating of a middle-third fracture of the clavicle

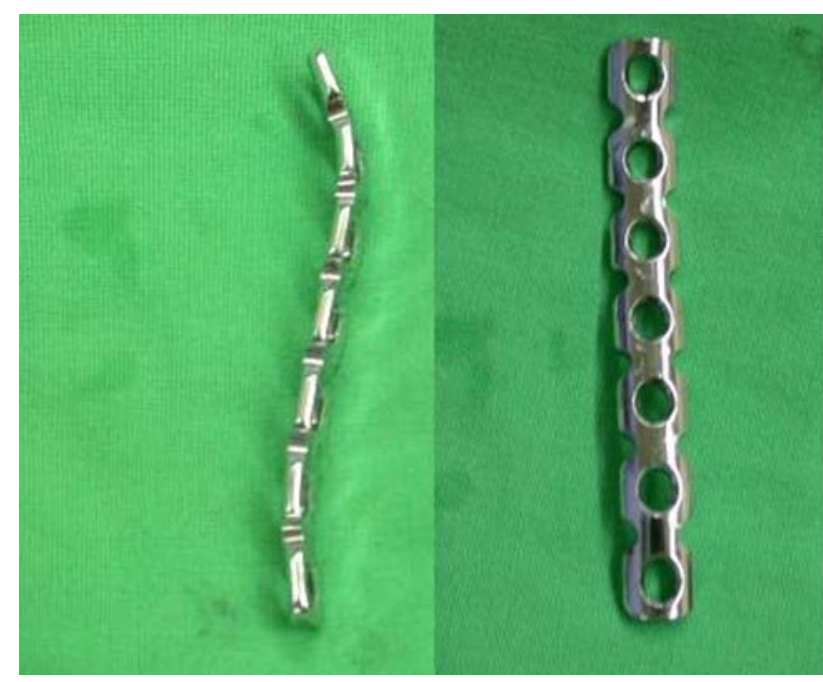

Fig. 3 Reconstruction plate prebent to match normal clavicle anatomy

4 weeks. At 5 weeks after surgery, unrestricted active and passive motion was initiated as tolerated, but lifting or resistance activities were prohibited for at least 8 weeks.
Following the discharge from hospital, the patients underwent follow-up at our outpatient clinic every 4 weeks. Fracture healing was assessed both clinically and radiographically at each follow-up visit. The functional results were evaluated at 6 months and 1 year, respectively. Any treatment complications were recorded. After bony union, patients underwent additional follow-up for a year.

\section{Results}

The mean follow-up period was 15 months (range 12-24 months). All of the fractures healed, as demonstrated both clinically and radiographically (Figs. 4, 5). The mean time to union was 14 weeks (range 8-20 weeks). At the time of latest follow-up, all of the patients had returned to their pre-injury activity level.

In ten patients, the plates were removed after the fractures healed. No patients required plate removal because of pain, prominence, cosmetic deformity or implant failure. No other complications were observed. No female patients complained of pain from bra straps, and no patients reported pain with backpack use or irritation from car seat belts.

\section{Discussion}

Most orthopedic surgeons prefer nonoperative treatment for nondisplaced middle-third fractures of the clavicle, using a sling or a figure 8 support. The optimal treatment modality for acute displaced middle-third fractures of the clavicle remains controversial, however [15]. Although most middle-third clavicle fractures unite uneventfully, conservative treatment has been found to be associated with higher rates of nonunion and patient dissatisfaction with the final result [6]. A prospective randomized controlled trial by the Canadian Orthopaedic Trauma Society compared plate and screw
Fig. 4 A 51-year-old man who sustained a middle-third fracture of the clavicle. a Anteroposterior radiographs of the shoulder showing displaced fracture of the right clavicle. b Radiograph of the shoulder showing anterior-inferior plating with a seven-hole $3.5-\mathrm{mm}$ reconstruction plate
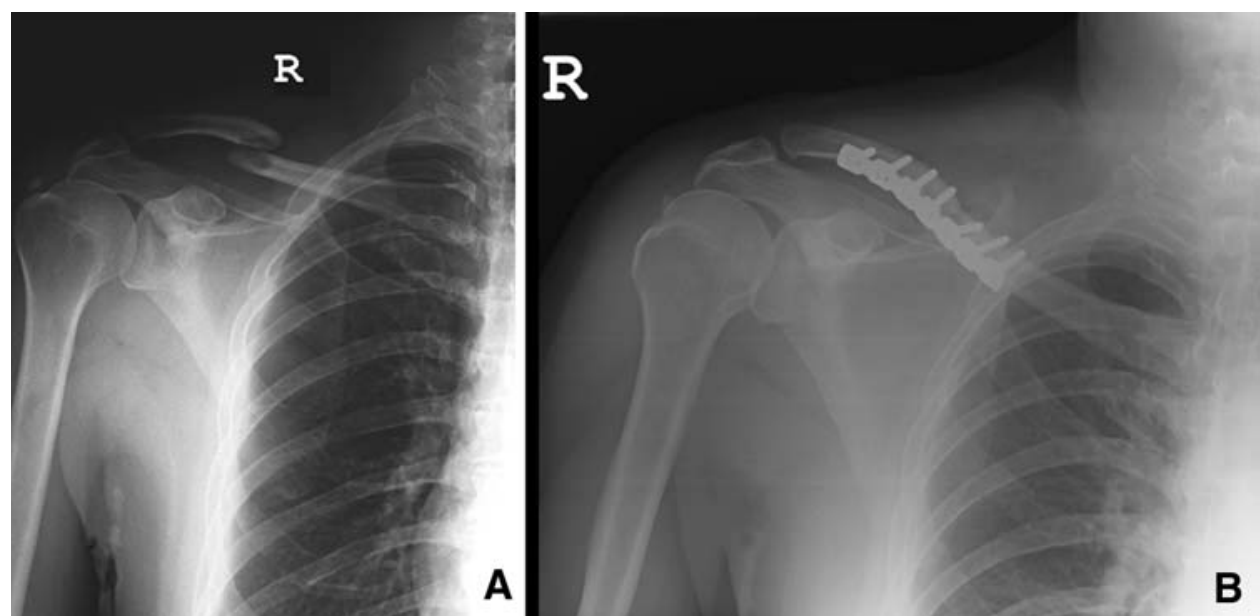
Fig. 5 A 60-year-old woman, who was involved in a traffic accident. a Radiograph of the right shoulder showing comminuted fracture of the middle third of the clavicle. $\mathbf{b}$ Radiograph of the right clavicle showing anterior-inferior plating with a reconstruction plate and multiple wiring
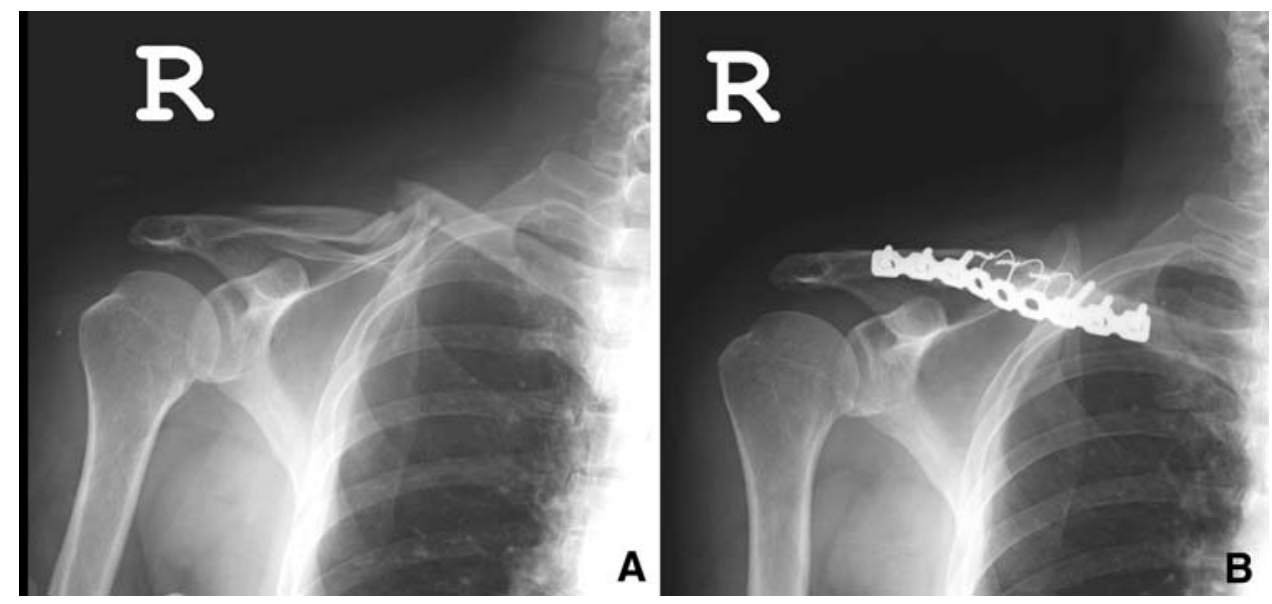

fixation with nonoperative treatment for displaced middlethird clavicle fractures [1]. The results showed that time to union was shorter in the operative group than in the nonoperative group; moreover, surgical fixation of a displaced clavicle fracture resulted in improved functional outcome and lower rates of malunion and nonunion compared with nonsurgical treatment after a year of follow-up. In this series, all fractures healed within 20 weeks, with an average healing time of 14 weeks. The longer healing time in some patients than in others may have been due to older patient age and fracture comminution.

Despite the popularity of plating for displaced clavicle fracture, optimal plate position is still debated. Jupiter and Leffert [8] suggested that superior plating was biomechanically better than inferior plating because the superior aspect of the clavicle was the load-bearing side. In superior plating, however, the downward force of the arm challenges the holding power of the screws in the often osteopenic lateral fragment, occasionally resulting in screw pullout. Kloen et al. [9] recommended plating on the anterior-inferior side because it functioned as an inferior buttress, especially under the lateral osteopenic bone, allowing the better medial fixation to support the construct without the risk of screw pullout of the lateral fragment.

In a biomechanical evaluation of clavicle fracture plating techniques, Celestre et al. [3] reported that repairing a middle-third clavicle fracture with a superior plate was better than anterior-inferior plating in terms of both load to failure and bending failure stiffness. In terms of axial compression stiffness, anterior-inferior plating was better than superior plating, but no statistically significant difference was found. Furthermore, locking plates offered significantly more stability in bending failure stiffness than nonlocking plates. Iannotti et al. [7] used a transverse osteotomy model to test the effects of plate location on the stability of middle-third clavicle fractures. They concluded that clavicles plated at the superior aspect exhibit significantly greater biomechanical stability than those plated at the anterior aspect, although no statistically significant difference in load to failure was found. Harnroongroj and Vanadurongwan [5] used a complex osteotomy model to create an unstable middle-third clavicle fracture. They found that anterior plating was significantly stronger than superior plating, but also that anterior plating failed before superior plating in a simple transverse osteotomy. The optimal position for plate fixation for middle-third clavicle fracture appears to depend on the fracture pattern. Thus, plating of the anterior-inferior surface of the clavicle does not appear to affect mechanical stability, but may affect the nature of complications and patient satisfaction. According to these results, anteriorinferior plating may be more appropriate than superior plating in the present study because most of the cases were unstable fractures.

Superior plating in the subcutaneous border of the clavicle has shown problems with implant prominence and local irritation over the implants. Hardware-related problems often necessitate implant removal. Shen et al. [14] reported that 171 of 232 reconstruction plates that had been applied to the superior surface of the clavicle were removed. Aside from the anticipated risks of a second surgical procedure, implant removal also carries the risk of refracture of the clavicle [2]. Anterior-inferior plating avoids these disadvantages of superior plating. Although the removal of the plates was not recommended in our patients, ten patients asked that the implants be removed after the fractures healed; six of these patients were worried about the effects of long-term plate retention, and the remaining four patients simply did not want the plates left in their bodies.

Anterior-inferior plating for middle-third fractures of the clavicle has the following advantages: (1) a $3.5-\mathrm{mm}$ reconstruction plate is easily contoured to the anterior-inferior surface of the clavicle and is strong enough to provide stable fixation leading to prompt bony union; (2) the screws are safely directed posterosuperiorly, away from the vulnerable infraclavicular structure; (3) the position allows for the placement of a longer screw for bone purchase, as the 
clavicle is wider than it is tall; (4) placing the plate on the anterior-inferior surface of the clavicle may reduce the risk of implant prominence. In our experience, although the clavicle is usually smaller in women than in men, in women its anteroposterior length is always greater than its superoinferior length; therefore, female patients are especially good candidates for this technique.

Collinge et al. [4] used an anterior-inferior plating technique similar to that used in this study to treat middle-third fractures and nonunion of the clavicle in 42 patients. They reported a 93\% success rate for acute fractures; all 16 patients with painful nonunion healed uneventfully after the index surgery using a $3.5-\mathrm{mm}$ reconstruction plate, dynamic compression plate, or fibular composite plate along with bone grafting. Kloen et al. [9] treated middlethird clavicle nonunion with anterior-inferior plating using a $3.5-\mathrm{mm}$ reconstruction plate and bone grafting. They reported a success rate of $100 \%$, with none of the 12 patients requiring further surgery or experiencing a loss of fixation.

All 26 patients in this study treated with $3.5-\mathrm{mm}$ reconstruction plates for acute middle-third clavicle fractures healed uneventfully. No patient reported problems with backpack use, discomfort from clothing worn about the shoulder or irritation from car seat belts, and no female patients had pain from bra straps. Scar formation was a concern for some patients treated with the plating technique, especially women. To minimize the cosmetic problems, 3 months of wound care using cosmetic strips was recommended. Although some patients used the cosmetic strips for only 1 month, none of the patients had unsightly scar formation at the time of the latest follow-up. In addition, the surgical wound was easy to cover with clothing, because the incision wound in anterior-inferior plating is a little lower than in superior plating. Although some patients complained of numbness inferior to the clavicle postoperatively, this condition progressively subsided. All of the patients in this series had excellent results.

The major limitation of this study is the lack of an alternative treatment or control group whose results could be compared with those of the treatment group. Although the sample size is small, the technique is easily reproducible by other orthopedic surgeons.

\section{Conclusion}

The results of this study show that anterior-inferior plating is an effective technique for the treatment of acute middle- third clavicle fractures in patients without multiple injuries. The technique provides stable fixation, avoids risk to the vital structures below the clavicle, and has a low rate of implant-prominence problems. Therefore, we recommend anterior-inferior plating for patients with acute middle-third clavicle fracture requiring internal fixation.

Open Access This article is distributed under the terms of the Creative Commons Attribution Noncommercial License which permits any noncommercial use, distribution, and reproduction in any medium, provided the original author(s) and source are credited.

\section{References}

1. Altamimi SA, McKee MD, Canadian Orthopaedic Trauma Society (2008) Nonoperative treatment compared with plate fixation of displaced midshaft clavicular fractures. J Bone Joint Surg Am 90(Suppl 2):1-8

2. Bostman O, Manninen M, Pihlajamaki H (1997) Complications of plate fixation in fresh displaced midclavicular fractures. J Trauma 43:778-783

3. Celestre P, Roberston C, Mahar A et al (2008) Biomechanical evaluation of clavicle fracture plating techniques: does a locking plate provide improved stability? J Orthop Trauma 22:241-247

4. Collinge C, Devinney S, Herscovici D et al (2006) Anteriorinferior plate fixation of middle-third fractures and nonunions of the clavicle. J Orthop Trauma 20:680-686

5. Harnroongroj T, Vanadurongwan V (1996) Biomechanical aspects of plating osteosynthesis of transverse clavicular fracture with and without inferior cortical defect. Clin Biomech 11:290-294

6. Hill JM, McGuire MH, Crosby LA (1997) Closed treatment of displaced middle-third fractures of the clavicle gives poor results. J Bone Joint Surg Br 79:537-539

7. Iannotti MR, Crosby LA, Stafford P et al (2002) Effect of plate location and selection on the stability of midshaft clavicle osteotomies: a biomechanical study. J Shoulder Elbow Surg 11:457-462

8. Jupiter JB, Leffert RD (1987) Nonunion of the clavicle. J Bone Joint Surg Am 69:753-760

9. Kloen P, Sorkin AT, Rubel IF et al (2002) Anteroinferior plating of midshaft clavicular nonunions. J Orthop Trauma 16:425-430

10. Marsh JL, Slongo TF, Agel J et al (2007) Fracture and dislocation classification compendium-2007. J Orthop Trauma 21:S1-133

11. Mueller M, Burger C, Florczyk A et al (2007) Elastic stable intramedullary nailing of midclaviclar fractures in adults: 32 patients followed for 1-5 years. Acta Orthop 78:421-423

12. Nordqvist A, Petersson C (1994) The incidence of fractures of the clavicle. Clin Orthop Relat Res 300:127-132

13. Nowak J, Holgersson M, Larsson S (2005) Sequelae from clavicular fractures are common. Acta Orthop 76:496-502

14. Shen WJ, Liu TJ, Shen YS (1999) Plate fixation of fresh displaced midshaft clavicle fractures. Injury 30:497-500

15. Zlowodzki M, Zelle BA, Cole PA et al (2005) Treatment of acute midshaft clavicle fractures: systemic review of 2144 fractures. J Orthop Trauma 19:504-507 